\title{
Edad, crecimiento y mortalidad natural de merluza de tres aletas, Micromesistius australis en el Océano Pacífico suroriental
}

\author{
Age, growth and natural mortality of southern blue whiting, Micromesistius australis \\ in the southeast Pacific Ocean \\ Mario Aguayo ${ }^{1}$, Javier Chong ${ }^{2}$ e Ignacio Payá ${ }^{3}$ \\ 1'Instituto de Investigación Pesquera, Casilla 350, Talcahuano, Chile
${ }^{2}$ Universidad Católica de la Santísima Concepción, Casilla 297, Concepción, Chile. jvchong@ucsc.cl
${ }^{3}$ Instituto de Fomento Pesquero, Casilla 8-V, Valparaíso, Chile
}

Abstract. - A study of age, growth and natural mortality of southern blue whiting, Micromesistius australis, was conducted using samples collected at latitudes from 46⿳⺈ to 56oS, between 1991 and 1995. The age determination was made by reading annual growth increments in sagitta otoliths. The lengths at different ages were estimated using corrected backcalculated lengths. The annual formation of one hyaline and opaque ring was determined by the analysis of the otolith margin. In the study of growth in length, the traditional models of von Bertalanffy (VBT), the generalized (VBG), and the Gompertz (GOMP) were used. The parameter estimations were performed using a nonlinear function, and maximum likelihood, for each sex and for both sexes pooled together. Based on the coefficient of determination, the sum of the squared residuals and the Akaike criterion (AIC), the most representative model of growth was the traditional von Bertalanffy. The comparison of growth parameters between the sexes by the Hotelling $\mathrm{T}^{2}$ test and the reason of least squares, indicate a significantly higher growth for females. The growth parameters estimated for Micromesistius australis in the southeastern Pacific are different from those recorded for the southwest Atlantic and New Zealand, as they belong to different stocks. The instantaneous coefficient of natural mortality (M) was estimated by empirical methods that consider the close relationship between $\mathrm{M}$ and the growth parameters. The estimated values are lower than those reported in previous studies.

Key words: Merlucciidae, otolith, Chilean coast

Resumen. - Se realizó un estudio de edad, crecimiento y mortalidad natural en merluza de tres aletas, Micromesistius australis, con muestras de especímenes capturados en el periodo 1991-1995 entre las latitudes 46S y 56ㅇ. La determinación de edad se efectuó mediante lectura de anillos crecimiento anual en los otolitos sagitta y las longitudes a distintas edades se estimaron mediante longitudes retrocalculadas corregidas. Se determinó la formación de un anillo hialino y uno opaco anual mediante el análisis estacional del borde. En el estudio del crecimiento en longitud se utilizaron los modelos de von Bertalanffy tradicional (VBT), el generalizado (VBG), y el de Gompertz (GOMP). La estimación de parámetros se realizó por sexo y total, utilizando un modelo no lineal. Sobre la base del coeficiente de determinación, suma de residuales al cuadrado y el criterio Akaike (AIC), el modelo más representativo del crecimiento fue el de von Bertalanffy tradicional. La comparación de parámetros de crecimiento entre sexos mediante el test $\mathrm{T}^{2}$ de Hotelling y la razón de mínimos cuadrados, indican para las hembras un crecimiento significativamente mayor que los machos. Los parámetros de crecimiento estimados para Micromesistius australis en el Pacífico suroriental son diferentes a los registrados para el Atlántico suroccidental y Nueva Zelanda, debido a que constituyen stocks distintos. El coeficiente instantáneo de mortalidad natural (M) fue estimado por métodos bioanalógicos o empíricos que consideran la interrelación entre M y los parámetros de crecimiento. Los valores estimados son menores a los informados en estudios anteriores.

Palabras clave: Merlucciidae, otolito, costa chilena

\section{INTRODUCCIÓN}

Micromesistius australis conocida en Chile con el nombre vernacular de "merluza de tres aletas", "polaca austral" en Argentina e internacionalmente como "southern blue whiting”, se distribuye en el cono sur de Sudamérica, desde la latitud $37^{\circ} \mathrm{S}$ a $\operatorname{los} 55^{\circ} \mathrm{S}$ en la plataforma argentina (Angelescu et al. 1958, Cassia 2000, Wöhler et al. 2007) y entre $\operatorname{los} 42^{\circ} \mathrm{S}$ y $57^{\circ} \mathrm{S}$ en la plataforma chilena, en profundidades de 20 a $800 \mathrm{~m}$ (Avilés et al. 1979, Cohen et 
al. 1990, Lillo et al. 2005). También se distribuye alrededor de las islas Georgias, Shetland del sur, Orcadas y sur de Nueva Zelanda.

Las capturas en el litoral chileno se iniciaron en 1978 como fauna acompañante en la pesquería de la merluza del sur (Merluccius australis Hutton), en la zona pesquera suraustral $\left(43^{\circ}-57^{\circ} \mathrm{S}\right)$, con un aumento gradual de las capturas (2.500 y 5.000 ton entre 1987 y 1992), hasta constituirse posteriormente como una especie objetivo con desembarques entre 20.000 y 25.000 ton, con un máximo de desembarque en 1998 de 40.587 ton (Aguayo et al. 1986, 1987, 1990, 1991, SERNAPESCA 2002).

Los primeros estudios del crecimiento de merluza de tres aletas en Chile corresponden a informes técnicos desarrollados por equipo de investigadores y técnicos especializados del Instituto de Fomento Pesquero (IFOP) y algunas universidades chilenas (Payá 1997, Ojeda et al. 1998). En ambos estudios se utilizaron otolitos sagitta y la longitud total a la edad se estimó por retrocálculo corregido (Francis 1990).

El presente trabajo tuvo como objetivos: i) Estimar los parámetros de la función de crecimiento en longitud y peso, y establecer si existe crecimiento diferencial entre sexos de merluza de tres aletas, ii) estimar la tasa instantánea de mortalidad natural. Esta información es básica para el conocimiento de la dinámica poblacional de merluza de tres aletas y por tanto es de gran relevancia para la evaluación de stock, y globalmente son antecedentes relevantes en la regulación pesquera anual de su pesquería.

\section{Material Y MÉtodos}

Ejemplares de Micromesistius australis fueron obtenidos en las capturas efectuadas por barcos industriales de arrastre, en el área de la pesquería demersal austral entre los años 1991 y 1995. En las muestras biológicas se registró la longitud total $(\mathrm{cm})$, el peso total $(\mathrm{g})$, y el sexo a cada ejemplar. Los otolitos sagitta fueron extraídos y guardados en seco en sobres rotulados con los respectivos datos y fecha de captura.

Para la determinación de la edad, se analizó una muestra de 4.348 ejemplares de merluza de tres aletas, correspondiendo 2.042 a hembras y 2.306 a machos*. El rango de longitud de las muestras estuvo entre 21 y $61 \mathrm{~cm}$ de longitud total.
Los otolitos sagitta se observaron con un microscopio estereoscópico con aumento 10x con micrómetro graduado en 100 divisiones $(1 \mathrm{div}=0,01 \mathrm{~mm})$ con luz reflejada y se registró el radio del otolito como la distancia desde el foco o punto origen hasta su borde caudal.

En la estimación de edad se contaron y midieron los radios de los anillos hialinos o translúcidos anuales o annuli desde el foco hasta el borde caudal de cada annulus (Chugunova 1963, Chilton \& Beamish 1982) y fueron hidratados $24 \mathrm{~h}$ antes de ser examinados. Luego, para resaltar los anillos de crecimiento, los otolitos fueron teñidos con tinta negra indeleble por su cara interna, y observados por su cara externa en una placa petri con agua corriente. El análisis siguiente consistió en determinar, el número de anillos hialinos (oscuros) y opacos (blanquecinos), registrar las medidas del radio del otolito (Ro) y el radio de cada anillo hialino ( $\mathrm{Rn}$ ), y determinar el tipo de borde. Previo a la medición de cada radio fue necesario pulir la cara interna de cada otolito con un esmeril de grano fino, a fin de visualizar el foco (Chong et al. 2007).

La validación de la periodicidad de la formación de los anillos anuales de crecimiento se realizó mediante el examen mensual del borde, utilizando la nomenclatura de Dannevig (1933), que consiste en asignar al borde del otolito la categoría de opaco ancho o angosto $(\mathrm{O}, \mathrm{o})$ y hialino ancho o angosto $(\mathrm{H}, \mathrm{h})$. La letra minúscula denota presencia incipiente del respectivo tipo de anillo en el borde.

\section{Crecimiento}

La estimación de longitudes a cada edad se efectuó por medio del retrocálculo, ya que debido al sesgo por la falta de juveniles (1-3 años) no fue posible la estimación de una curva representativa del crecimiento. En el retrocálculo o determinación de longitudes a edades pretéritas se corrigen las desviaciones que se producen al calcular directamente la longitud del pez, mediante la ecuación de regresión lineal entre el radio del otolito y la longitud del pez (Francis 1990). Esta corrección integra el radio del otolito ( $\mathrm{Rc})$, el anillo de crecimiento respectivo $(\mathrm{Ri})$, la longitud del pez al momento de la captura (Lc) y las constantes de la regresión que entreguen el mejor ajuste de la relación entre el incremento en longitud del pez y el radio del otolito. La estimación de las longitudes promedios a cada edad, mediante este método, involucra el retrocálculo para cada anillo hialino anual (Araya \& Cubillos 2002).

* Muestras obtenidas por IFOP y analizadas en el marco del proyecto FIP 97-15. 
La relación longitud total del pez versus el radio del otolito se determinó a través del método de regresión entre los valores de longitud total y radio otolito, la cual puede ser lineal, potencial u otra, y seleccionando en forma preliminar aquella que presente un mejor ajuste $\left(>r^{2}\right)$. Cuando la relación entre la longitud del pez y el radio del otolito corresponde a una función lineal, la ecuación de retrocálculo corresponde a:

$$
L_{i}=\left[\frac{c+d R_{i}}{c+d R_{c}}\right] \cdot L_{c}
$$

Cuando la relación entre el tamaño del pez y el tamaño del otolito no es lineal, la expresión más comúnmente usada para el retrocálculo es (Araya \& Cubillos 2002):

$$
L_{i}=u \cdot R_{i}^{v}
$$

Donde $u$ y $v$, intercepto y pendiente de regresión potencial.

La función de retrocálculo corregida es:

$$
L_{i}=\left(\frac{R_{i}}{R_{c}}\right)^{v} \cdot L_{c}
$$

Para determinar el modelo de crecimiento que presentaba el mejor ajuste a los datos observados de longitud-edad, se utilizaron los modelos de von Bertalanffy tradicional (VBT), von Bertalanffy generalizado (VBG) y Gompertz (GOMP), cuyas expresiones son respectivamente:

$$
\begin{aligned}
& \hat{L}_{t}=L_{\infty} *\left(1-e^{-K\left(t-t_{0}\right)}\right)+\varepsilon_{t} \\
& \hat{L}_{t}=L_{\infty} *\left(1-e^{-K\left(t-t_{0}\right)}\right)^{D}+\varepsilon_{t} \\
& \left.\hat{L}_{t}=L_{\infty} * e^{-e^{-K\left(t-t^{*}\right)}}\right)+\varepsilon
\end{aligned}
$$

Donde:

$\mathrm{L}_{\mathrm{t}}$ : longitud total a edad $\mathrm{t}$

$\mathrm{L}_{\propto}$ : longitud máxima promedio

$\mathrm{K}$ : coeficiente de crecimiento

$\mathrm{t}_{0}$ : edad teórica a longitud cero

D : parámetro de crecimiento alométrico

t* : edad en punto de inflexión de curva

$\varepsilon_{\mathrm{t}}$ : error asociado a la estimación de los parámetros
En general, en estudios de crecimiento en peces el modelo de crecimiento más utilizado, tanto en longitud como en peso corresponde al de VBT, cuya expresión integrada y linealizada ha sido propuesta por Beverton \& Holt (1957) e incluye un factor de error aditivo $\left(\varepsilon_{t}\right)$.

El no contar con información directa de peso medio a la edad, imposibilitó ajustar una curva de crecimiento en peso con sus correspondientes parámetros. Por esta razón se estimó un peso asintótico a partir de la longitud asintótica y la relación longitud-peso, para el periodo 1991-1995:

$$
W_{\infty}=a *\left(L_{\infty}\right)
$$

Los parámetros de crecimiento $\mathrm{K}$ y $\mathrm{t}_{0}$ corresponden a los de crecimiento en longitud, luego la expresión de crecimiento en peso de von Bertalanffy es:

$$
\hat{W}_{t}=W_{\infty} *\left(1-e^{-K\left(t-t_{0}\right)}\right)^{b}+\varepsilon_{t}
$$

El modelo de crecimiento se ajustó a los datos de edad y longitud individuales mediante ajuste no lineal. El procedimiento de minimización de cuadrados tiene la expresión:

$$
\mathrm{SCR}=\sum_{i=1}^{n}\left[L t-L_{\infty}\left(1-e^{-\mathrm{k}(\mathrm{t}-\mathrm{t} 0)}\right)\right]^{2}
$$

El proceso de ajuste es iterativo y se consideró como valores iniciales los parámetros de crecimiento estimados en forma específica para cada sexo por Ojeda et al.(1998).

Para comprobar si existían diferencias significativas entre las funciones de crecimiento de machos y hembras, se utilizó el test $\mathrm{T}^{2}$ de Hotelling (Bernard 1981), pero con la formulación propuesta por Cerrato (1990) de acuerdo a la siguiente expresión:

$$
T^{2}=\frac{\left(P_{1}-P_{2}\right)^{\prime}}{S_{p 1-p 2}^{-1}\left(P_{1}-P_{2}\right)}
$$

Donde:

$\left(\mathrm{P}_{1}-\mathrm{P}_{2}\right)$ : vector diferencia de parámetros

$\left(\mathrm{P}_{1}-\mathrm{P}_{2}\right)^{\prime}$ : vector transpuesto del anterior

$S_{p 1-p 2}^{-1}:$ matriz de varianza-covarianza del vector diferencia de parámetros

Considerando " $\mathrm{H}_{0}$ : Igualdad de vectores de los parámetros de crecimiento entre los sexos", la hipótesis $\mathrm{H}_{0}$ es aceptada si el $\mathrm{T}^{2}$ calculado, es menor al valor crítico $\mathrm{T}_{0}{ }^{2}$, obtenido mediante la expresión: 


$$
T_{0}^{2}=3 \frac{\left(N_{1}+N_{2}-6\right)}{\left(N_{1}+N_{2}-8\right)} F_{\alpha}\left(3, N_{1}+N_{2}-6\right)
$$

Donde $\mathrm{F}$ corresponde el percentil de la distribución de Fisher con 3 y $\mathrm{N}_{1}+\mathrm{N}_{2}-6$ grados de libertad. En este caso, la hipótesis se rechaza cuando $\mathrm{T}^{2}$ calculado $>\mathrm{T}_{0}{ }^{2}$ (crítico)

Un segundo método de comparación para confrontar resultados fue el análisis de la suma de cuadrados residuales sugerida por Chen et al. (1992), cuya formulación es la siguiente:

$$
F=\frac{\frac{S C R_{p}-S C R_{s}}{3(K-1)}}{\frac{S C R_{s}}{N-3 K}}
$$

Donde:

$\mathrm{N}$ : número total de muestras

K: número de grupos en comparación

$\mathrm{SCR}_{s}$ : suma de cuadrados residuales de ambos grupos (machos más hembras)

$\mathrm{SCR}_{p}$ : suma de cuadrados residuales del ajuste agrupado (sin diferenciar por sexo)

En la elección del modelo más representativo del crecimiento de merluza de tres aletas, también se consideró la suma de residuales al cuadrado (SRC) y el coeficiente de determinación $\mathrm{r}^{2}$ que mide la proporción de la variabilidad total de la variable dependiente $\left(\mathrm{L}_{\mathrm{t}}\right)$ que es explicada la variación de la variable independiente (t). Además, se utilizó el criterio de información de Akaike o AIC (1974) que aún cuando no es un test estadístico, es una medida de la bondad de ajuste de un modelo estadístico estimado. Este criterio selecciona el modelo que mejor explica los datos con un mínimo de parámetros libres. En definitiva el modelo seleccionado es aquel que presenta un menor valor. $\mathrm{Su}$ forma general es:

$$
A I C=2 k-2 \operatorname{Ln}(L)
$$

Donde $\mathrm{k}$ es el número de parámetros y L es el valor de la función de máxima verosimilitud para el modelo estimado y cuya expresión es:

$$
\operatorname{Li}=1 / 2 * \operatorname{Ln}\left(\sigma^{2}\right)+\left(\operatorname{SSRi} /\left(2 *\left(\sigma^{2}\right)\right)\right)
$$

\section{Tasa INSTANTÁNEA DE MORTALIDAD NATURAL}

La tasa instantánea de mortalidad natural $\mathrm{M}$ es la tasa de muerte producida por cualquier causa diferente a la pesca, es alta en las edades jóvenes y tiende a estabilizarse en edades mayores. En la estimación de la tasa instantánea de mortalidad natural se utilizaron métodos bioanalógicos o empíricos que consideran valores de parámetros de crecimiento y ambientales. En el caso de estos métodos, las fórmulas analíticas provienen de relaciones teóricas entre los diferentes parámetros, o bien, se deriva de regresiones entre $\mathrm{M}$ y uno o más parámetros de crecimiento. Estos modelos tienen como principal ventaja el que requieren una mínima cantidad de datos (Sparre \& Venema 1998).

El método de Pauly (1980) proporciona un estimado de M sobre la base de la relación que existe entre este parámetro y los parámetros de crecimiento, en especial, el parámetro $\mathrm{K}$ de la ecuación de crecimiento de VBT y la temperatura del hábitat de la especie, por el efecto que tiene sobre el coeficiente $\mathrm{K}$ y la longitud asintótica $\left(\mathrm{L}_{\infty}\right)$. Se consideró una temperatura $(\mathrm{T})$ del hábitat de $7^{\circ} \mathrm{C}$ (Ojeda et al. 1998). La ecuación que representa esta relación es la siguiente:

$\operatorname{Ln} \mathrm{M}=-0,0152-0,279 \times \ln \mathrm{L}_{\infty}+0,6543 \times \ln \mathrm{K}+0,4634 \times \ln \mathrm{T}$

El método de Alverson \& Cartney (1975) estima M a partir de la siguiente fórmula:

$$
M=\frac{b K}{\left[\exp \left(K\left(t^{*}\right)-1\right)\right]}
$$

Donde $\mathrm{b}$ es el exponente isométrico de la relación longitud-peso $(\mathrm{b}=3)$ y $\mathrm{K} \mathrm{y}_{0}$ son los parámetros de la ecuación de crecimiento de VBT. Por otra parte, la edad crítica $\left(\mathrm{t}^{*}\right)$ que corresponde a la edad de biomasa máxima de una cohorte, se calcula de acuerdo con Csirke (1980) mediante la expresión:

$$
t^{*}=t o+\frac{1}{K} * \ln \left[\frac{3 K}{M}+1\right]
$$

Si $\mathrm{t}_{0}$ se ignora, entonces,

$$
t^{*}=\frac{1}{K} * \ln \left[\frac{3 K}{M}+1\right]
$$

En el método de Höening (1983), el autor encontró una relación empírica entre la tasa instantánea de mortalidad total $(\mathrm{Z})$ y la edad máxima $\left(\mathrm{T}_{\max }\right)$ observada y determinó 
valores de $\mathrm{a}=1,46 \mathrm{y} \mathrm{b}={ }^{-} 1,01$ para peces sobre la base de 84 stocks de 53 especies diferentes. En general, estos stocks son vírgenes o de explotación incipiente, de manera que $\mathrm{Z}$ es una estimación de M. La edad máxima se estimó mediante la expresión de Taylor (1960), quien señala que la edad máxima teórica $\left(\mathrm{T}_{\mathrm{m}}\right)$ ocurriría cuando el $95 \%$ de la cohorte alcanza la longitud asintótica, i.e.,

$$
\ln (Z)=a+b \cdot \ln \left(T_{m}\right) \quad \text { y } \quad T_{m}=t_{0}+\frac{3}{K}
$$

Donde $\mathrm{t}_{0}$ y $\mathrm{K}$ son parámetros de crecimiento del modelo de von Bertalanffy y $\mathrm{T}_{\mathrm{m}}$ se consideró como estimador de $\mathrm{T}_{\max }$.

Para estimar la variabilidad de los estimados de $\mathrm{M}$, se consideró que en general, los métodos empíricos no entregan estimados de varianza, razón por la cual se procedió a realizar un remuestreo mediante un bootstrap (Efron 1985), el que fue evaluado numéricamente por medio de ensayos de Monte Carlo, que básicamente consistió en generar muestras aleatorias desde una distribución de probabilidades conocidas. La variabilidad se midió en términos de la desviación estándar del análisis de Monte Carlo y para asegurar la estabilidad de los estimados, se consideraron 3000 muestras, utilizando números aleatorios. La desviación estándar se determinó como:

$$
\text { d.e. }(\mathrm{M})=\sqrt{\frac{\sum_{i=1}^{m}\left(M i-\bar{M}^{2}\right.}{m-1}}
$$

Donde $\mathrm{m}$ es el número de muestras (3000) y $M i$ es la mortalidad natural de la muestra $i$ y la mortalidad natural promedio es:

$$
\bar{M}=\frac{\sum_{i=1}^{m} M i}{m}
$$

En la obtención de las muestras aleatorias de M, se emplearon los errores estándares de los parámetros de las ecuaciones de los diferentes métodos citados. Luego de obtener los estimados de M y sus errores estándares mediante los diferentes métodos, se calculó un valor de $\mathbf{M}$ promedio, ponderado por el inverso de la varianza de cada estimado:

$$
\widehat{M}=\frac{\sum_{j=1}^{n} w j M j}{\sum_{j=1}^{n} w j}
$$

Donde $M_{j}$ corresponde a la estimación de mortalidad natural por el método $j(=1,2 \ldots, \mathrm{n})$ y $w_{j}$ es el inverso de la varianza $\left(w_{j}=1 / S^{2}\right)$

\section{Resultados}

El número máximo de annuli observados en los otolitos de merluza de tres aletas fue 18 , tanto en machos como en hembras. En la Fig. 1 se presenta una fotografía de un otolito sagitta iluminado con luz incidente que corresponde un

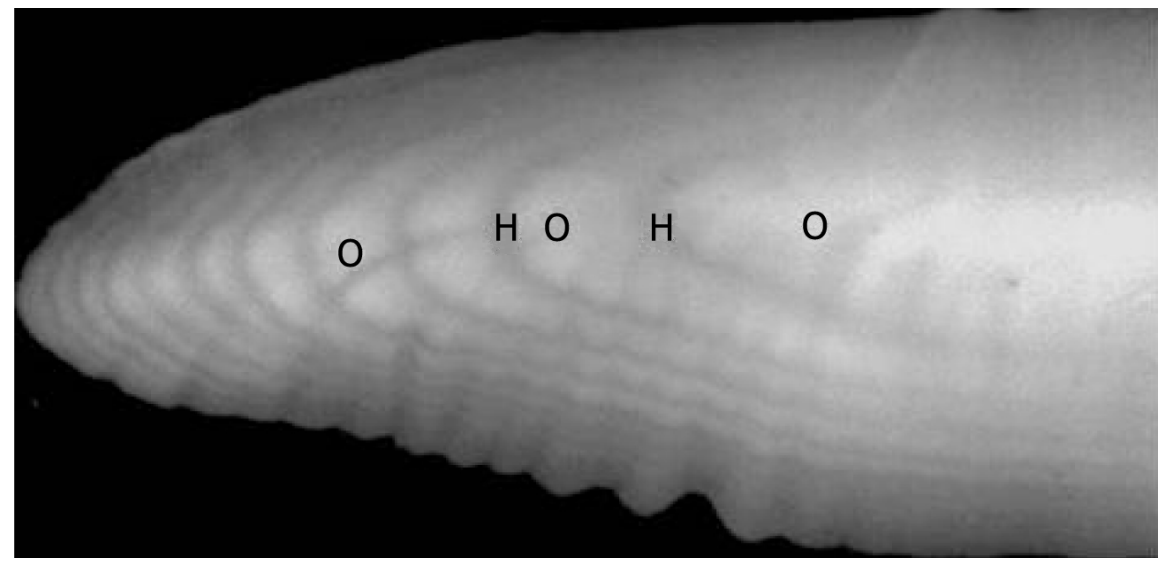

Figura 1. Fotografía de otolito sagitta de $M$. australis de 11 años y $53 \mathrm{~cm}$ ( $\mathrm{H}=$ anillos hialinos, $\mathbf{O}=$ anillos opacos) / Sagittal otolith photograph of 11 years and $53 \mathrm{~cm}$ in $M$. australis ( $H$ =hyaline rings, $0=$ opaque rings) 
Tabla 1. Parámetros de la regresión longitud total-radio otolito en M. australis por año y sexo / Parameters of the total length-otolith radius regression of $M$. australis by year and sex

\begin{tabular}{|c|c|c|c|c|c|c|c|}
\hline Año & Sexo & $\begin{array}{l}\text { Coeficientes } \\
\text { regresión }\end{array}$ & $\begin{array}{c}\text { Valor } \\
\text { estimado }\end{array}$ & $\begin{array}{l}\text { Error } \\
\text { estándar }\end{array}$ & L. Inf. $95 \%$ & L. Sup. $95 \%$ & $r^{2}$ \\
\hline \multirow[t]{4}{*}{1990} & macho & u & 0,54955 & 0,05751 & 0,42804 & 0,65386 & 0,742 \\
\hline & & $\mathrm{v}$ & 0,95521 & 0,02267 & 0,91070 & 0,99973 & \\
\hline & hembra & $\mathrm{u}$ & 0,59022 & 0,06479 & 0,46290 & 0,71754 & 0,796 \\
\hline & & $\mathrm{v}$ & 0,94086 & 0,02319 & 0,89528 & 0,98643 & \\
\hline \multirow[t]{4}{*}{1991} & macho & $\mathrm{u}$ & 0,69948 & 0,07748 & 0,54725 & 0,85171 & 0,757 \\
\hline & & $\mathrm{v}$ & 0,90182 & 0,02365 & 0,85535 & 0,94829 & \\
\hline & hembra & $\mathrm{u}$ & 0,23465 & 0,02572 & 0,18414 & 0,28517 & 0,818 \\
\hline & & $\mathrm{v}$ & 1,13823 & 0,02338 & 1,09230 & 1,18416 & \\
\hline \multirow[t]{4}{*}{1992} & macho & $\mathrm{u}$ & 0,40674 & 0,05900 & 0,29063 & 0,52284 & 0,807 \\
\hline & & $\mathrm{v}$ & 1,01991 & 0,03107 & 0,95876 & 1,08106 & \\
\hline & hembra & $\mathrm{u}$ & 0,30213 & 0,04958 & 0,20444 & 0,39983 & 0,836 \\
\hline & & $\mathrm{v}$ & 1,08498 & 0,03502 & 1,01598 & 1,15399 & \\
\hline \multirow[t]{4}{*}{1993} & macho & $\mathrm{u}$ & 0,30089 & 0,03403 & 0,23405 & 0,36773 & 0,802 \\
\hline & & $\mathrm{v}$ & 1,07998 & 0,02404 & 1,03276 & 1,12720 & \\
\hline & hembra & $\mathrm{u}$ & 0,34637 & 0,04151 & 0,26483 & 0,42791 & 0,784 \\
\hline & & $\mathrm{v}$ & 1,05616 & 0,02528 & 1,00648 & 1,10583 & \\
\hline \multirow[t]{4}{*}{1994} & macho & $\mathrm{u}$ & 0,54782 & 0,06210 & 0,42585 & 0,66978 & 0,740 \\
\hline & & $\mathrm{v}$ & 0,94531 & 0,02411 & 0,89796 & 0,99267 & \\
\hline & hembra & $\mathrm{u}$ & 0,29135 & 0,03322 & 0,22608 & 0,35663 & 0,819 \\
\hline & & $\mathrm{v}$ & 1,08622 & 0,02415 & 1,03877 & 1,13368 & \\
\hline \multirow[t]{4}{*}{1995} & macho & $\mathrm{u}$ & 0,21604 & 0,03525 & 0,14666 & 0,28542 & 0,796 \\
\hline & & $\mathrm{v}$ & 1,14659 & 0,03481 & 1,07807 & 1,21511 & \\
\hline & hembra & u & 0,23953 & 0,05739 & 0,12614 & 0,35293 & 0,770 \\
\hline & & $\mathrm{v}$ & 1,13034 & 0,05116 & 1,02926 & 1,23142 & \\
\hline \multirow[t]{4}{*}{$1990-95$} & macho & $\mathrm{u}$ & 0,50126 & 0,02270 & 0,45674 & 0,54578 & 0,739 \\
\hline & & $\mathrm{v}$ & 0,96900 & 0,00964 & 0,95009 & 0,98790 & \\
\hline & hembra & u & 0,32698 & 0,01555 & 0,29648 & 0,35748 & 0,803 \\
\hline & & $\mathrm{v}$ & 1,06524 & 0,01008 & 1,04546 & 1,08501 & \\
\hline \multirow[t]{2}{*}{$1990-95$} & ambos & $\mathrm{u}$ & 0,38776 & 0,01301 & 0,36225 & 0,41327 & 0,765 \\
\hline & & $\mathrm{v}$ & 1,02607 & 0,00713 & 1,01209 & 1,04005 & \\
\hline
\end{tabular}

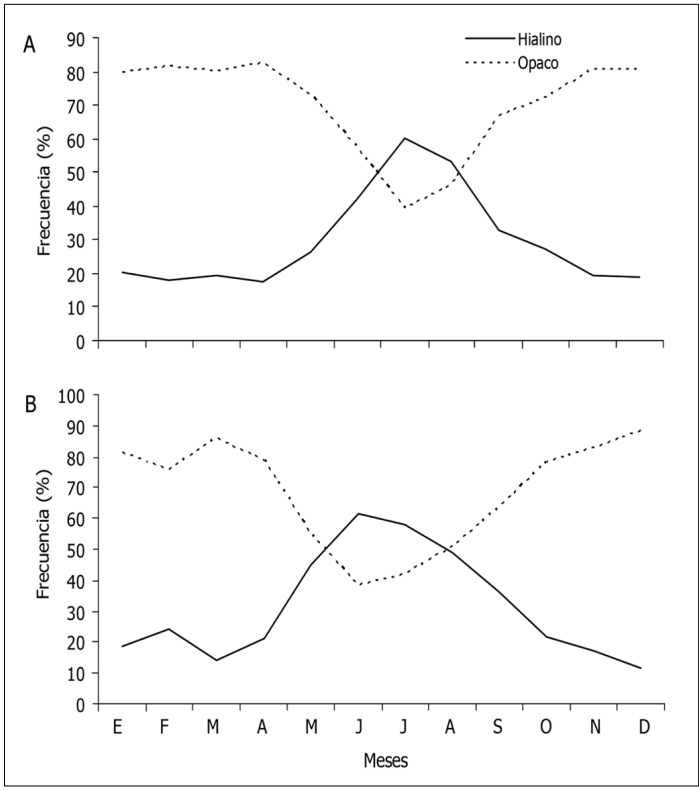

Figura 2. Frecuencia mensual de bordes hialinos y opacos en los otolitos sagitta de M. australis. A) machos y B) hembras / Monthly frequency of hyaline and opaque edges in the sagittal otoliths of $M$. australis. A) male and B) females ejemplar de $53 \mathrm{~cm}$ con 11 años de edad, donde se puede observar en forma nítida los annuli o anillos hialinos $(\mathrm{H})$ de crecimiento lento, alternados con anillos opacos $(\mathrm{O})$ de crecimiento rápido.

\section{DETERMINACIÓN DE LA EDAD}

La determinación de edad se efectuó mediante el registro de anillos hialinos anuales validados mediante la formación estacional de los anillos en el borde de los otolitos como se describe a continuación.

En cuanto a la periodicidad en la formación de los anillos de crecimiento, la distribución mensual (\%) de los bordes en los otolitos muestra que las mayores frecuencias de bordes hialinos se presentan entre junio y agosto con un máximo en julio en los machos y entre mayo y julio con un máximo en junio en las hembras. Por otra parte, el borde opaco predomina durante los meses de noviembre hasta abril con valores máximos alrededor de $80 \%$ (Fig. 2). Esto señala la formación de un anillo hialino y uno opaco anual.

Las relaciones potenciales ajustadas por sexo en forma no lineal entre la longitud total del pez y el radio del otolito, 
Tabla 2. Longitud total retrocalculada corregida para machos de M. australis / Back calculated total length corrected for M. australis males

\begin{tabular}{lrrrrrrrr}
\hline $\begin{array}{l}\text { Longitud } \\
\text { retrocalculada }\end{array}$ & 1990 & 1991 & 1992 & 1993 & 1994 & 1995 & $\mathrm{~N}$ & $\begin{array}{c}\text { Promedio } \\
\text { ponderado }\end{array}$ \\
\hline L1 & 26,6 & 27,4 & 25,3 & 25,0 & 26,3 & 23,1 & 2423 & 25,8 \\
L2 & 33,9 & 34,2 & 32,4 & 32,2 & 33,3 & 30,9 & 2894 & 33,1 \\
L3 & 37,7 & 38,4 & 37,1 & 36,8 & 37,3 & 35,6 & 2818 & 37,3 \\
L4 & 40,7 & 41,3 & 40,4 & 40,1 & 40,1 & 38,8 & 2680 & 40,3 \\
L5 & 42,9 & 43,5 & 42,8 & 42,7 & 42,3 & 41,7 & 2444 & 42,7 \\
L6 & 44,8 & 45,2 & 44,8 & 44,8 & 44,0 & 44,0 & 2217 & 44,6 \\
L7 & 46,2 & 46,6 & 46,5 & 46,5 & 45,2 & 45,7 & 1990 & 46,1 \\
L8 & 47,6 & 47,9 & 47,9 & 47,8 & 46,2 & 47,1 & 1534 & 47,3 \\
L9 & 48,7 & 49,4 & 49,2 & 48,7 & 47,1 & 47,9 & 1147 & 48,3 \\
L10 & 49,6 & 50,1 & 50,5 & 49,5 & 47,9 & 48,6 & 855 & 49,1 \\
L11 & 50,2 & 51,2 & 50,9 & 50,6 & 48,6 & 49,5 & 625 & 49,9 \\
L12 & 51,2 & 51,8 & 52,3 & 51,5 & 49,3 & 50,0 & 392 & 50,8 \\
L13 & 52,3 & 52,7 & 53,1 & 52,3 & 50,7 & 50,6 & 219 & 52,0 \\
L14 & 52,7 & 53,3 & 54,4 & 53,2 & 49,7 & 52,3 & 116 & 52,8 \\
L15 & 53,6 & 53,6 & 55,6 & 53,6 & 50,1 & & 61 & 53,5 \\
L16 & 53,3 & 52,6 & 53,3 & 53,6 & & & 27 & 53,2 \\
L17 & 52,9 & 54,2 & 54,0 & 54,0 & & & 12 & 53,6 \\
L18 & 53,7 & 51,4 & & & & & 4 & \\
\hline
\end{tabular}

presentaron un mejor ajuste que la relación lineal y los valores de los parámetros se entregan en la Tabla 1. La Fig. 3 representa la relación entre longitud total del pez y el radio del otolito por sexo, para el periodo 1991-1995. El incremento en tamaño del radio del otolito es similar en hembras y machos hasta los $44 \mathrm{~cm}$ de longitud total, a partir de cuyo valor el aumento en tamaño de los otolitos de las hembras es mayor.

\section{Estimación DE CRECIMIENTO}

Para la estimación de crecimiento en longitud mediante retrocálculo o edades pretéritas el número de longitudes retrocalculadas corregidas en machos fue de 22.458 y para hembras de 18.408. En general, se observó una baja presencia de ejemplares en edades superiores a los 12 años, en ambos sexos.

Las longitudes promedios retrocalculadas para los machos y las hembras son muy similares hasta la edad 4, presentando diferencias en el crecimiento desde la edad 5, alcanzando las hembras mayores longitudes a las respectivas edades (Tablas 2 y 3 ).

En la Tabla 4 se entregan los parámetros de crecimiento ajustados, para los métodos de VBT, VBG y GOMP utilizando los datos individuales de las longitudes retrocalculadas. En esta tabla se puede observar que considerando el coeficiente de determinación $\left(r^{2}\right)$, la suma de residuales al cuadrado (SRC) y el criterio Akaike (1974) (AIC), los modelos que presentan un mejor ajuste son los de VBT y dado que habitualmente el modelo tradicional es
Tabla 3. Longitud total retrocalculada corregida para hembras de M. australis / Back calculated total length corrected for M. australis females

\begin{tabular}{lrrrrrrrr}
\hline $\begin{array}{l}\text { Longitud } \\
\text { retrocalculada }\end{array}$ & 1990 & 1991 & 1992 & 1993 & 1994 & 1995 & $\mathrm{~N}$ & $\begin{array}{c}\text { Promedio } \\
\text { ponderado }\end{array}$ \\
\hline L1 & & & & & & & \\
L2 & 37,5 & 24,3 & 25,0 & 26,1 & 25,2 & 24,0 & 1997 & 25,4 \\
L3 & 39,0 & 37,1 & 32,4 & 33,6 & 32,9 & 32,2 & 2371 & 33,0 \\
L4 & 42,3 & 40,7 & 41,2 & 42,1 & 42,4 & 40,8 & 2154 & 41,4 \\
L5 & 44,8 & 43,3 & 43,9 & 44,8 & 44,1 & 43,7 & 1975 & 43,8 \\
L6 & 46,8 & 45,5 & 46,1 & 46,9 & 46,2 & 46,3 & 1788 & 46,0 \\
L7 & 48,0 & 47,3 & 48,1 & 48,7 & 47,9 & 48,1 & 1549 & 47,7 \\
L8 & 49,8 & 48,8 & 49,7 & 50,3 & 49,1 & 49,7 & 1251 & 49,1 \\
L9 & 51,2 & 50,0 & 50,9 & 51,6 & 49,8 & 50,8 & 967 & 50,2 \\
L10 & 52,6 & 51,6 & 51,7 & 52,7 & 50,8 & 51,6 & 709 & 51,4 \\
L11 & 53,4 & 52,9 & 52,4 & 53,8 & 51,9 & 52,2 & 543 & 52,3 \\
L12 & 54,3 & 53,9 & 53,1 & 55,3 & 53,0 & 53,5 & 347 & 53,4 \\
L13 & 55,1 & 55,1 & 53,8 & 56,1 & 53,8 & 54,5 & 229 & 54,3 \\
L14 & 55,2 & 56,1 & 54,8 & 56,6 & 54,6 & & 118 & 55,2 \\
L15 & 55,3 & 56,2 & 55,8 & 57,0 & 53,6 & & 67 & 55,3 \\
L16 & 55,8 & 56,7 & 54,0 & 57,7 & & & 39 & 56,0 \\
L17 & 55,9 & 58,7 & 54,5 & 58,9 & & & 19 & \\
L18 & 58,9 & 58,2 & & & & & 9 & \\
\hline
\end{tabular}

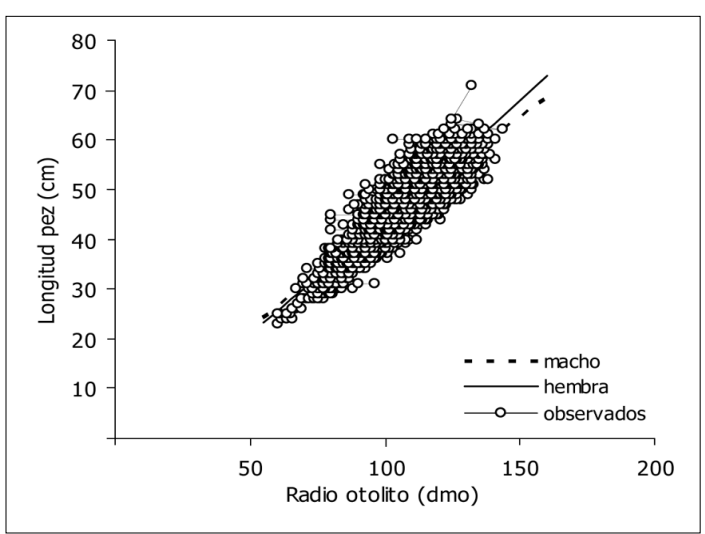

Figura 3. Relación potencial entre la longitud total y el radio del otolito para machos y hembras de M. australis / Power relationship between total length and otolith radius for males and females of $M$. australis.

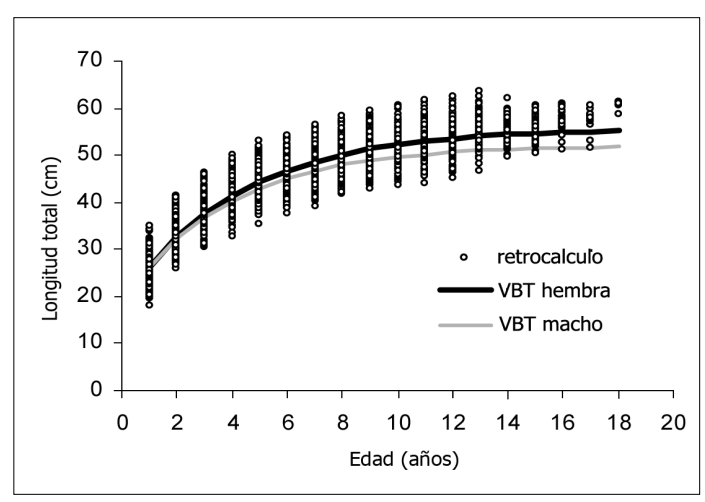

Figura 4. Curvas de crecimiento en longitud ajustadas para $M$. australis / Growth curves in length fitted for M. australis

Vol. 45, S1, $2010-729$ 
Tabla 4. Parámetros de crecimiento en longitud para machos y hembras de $M$. australis. Error estándar entre paréntesis. (VBT, von Bertalanffy tradicional; VBG, von Bertalanffy generalizado; GOMP, Gomperzt; t*, punto de inflexión; D, parámetro de alometría) / Growth length parameters for males and females of M. australis. Standard error in parentheses. (VBT, von Bertalanffy traditional; VBG von Bertalanffy generalized; GOMP, Gomperzt; t*, inflection point; D, allometry parameter)

\begin{tabular}{|c|c|c|c|c|c|c|c|c|c|}
\hline Sexo & Modelo & $\mathrm{L}_{\infty}$ & $\mathrm{K}$ & $\mathrm{t}_{0}$ & $t^{*}$ & $\mathrm{D}$ & $\mathrm{r}^{2}$ & SCR & AIC \\
\hline \multirow[t]{3}{*}{ Hembras } & VBT & $\begin{array}{c}55,7 \\
(0,096)\end{array}$ & $\begin{array}{c}0,239 \\
(0,002)\end{array}$ & $\begin{array}{l}-1,679 \\
(0,021)\end{array}$ & & & 0,92 & 84839 & 13,81 \\
\hline & VBG & $\begin{array}{c}62,9 \\
(0,604)\end{array}$ & $\begin{array}{c}0,085 \\
(0,005)\end{array}$ & $\begin{array}{c}0,270 \\
(0,029)\end{array}$ & & $\begin{array}{l}0,320 \\
0,007\end{array}$ & 0,93 & 80647 & 13,77 \\
\hline & GOMP & $\begin{array}{c}54,5 \\
(0,079)\end{array}$ & $\begin{array}{c}0,313 \\
(0,002)\end{array}$ & & $\begin{array}{l}-0,087 \\
(0,011)\end{array}$ & & 0,92 & 84839 & 13,84 \\
\hline \multirow[t]{3}{*}{ Machos } & VBT & $\begin{array}{c}52,1 \\
(0,080)\end{array}$ & $\begin{array}{c}0,262 \\
(0,002)\end{array}$ & $\begin{array}{l}-1,685 \\
(0,021)\end{array}$ & & & 0,90 & 102508 & 14,20 \\
\hline & VBG & $\begin{array}{c}57,2 \\
(0,419)\end{array}$ & $\begin{array}{c}0,105 \\
(0,006)\end{array}$ & $\begin{array}{c}0,255 \\
(0,030)\end{array}$ & & $\begin{array}{c}0,309 \\
(0,008)\end{array}$ & 0,91 & 98762 & 14,77 \\
\hline & GOMP & $\begin{array}{c}51,2 \\
(0,067)\end{array}$ & $\begin{array}{c}0,335 \\
(0,002)\end{array}$ & & $\begin{array}{l}-0,276 \\
(0,012)\end{array}$ & & 0,90 & 105968 & 14,22 \\
\hline \multirow[t]{3}{*}{$\begin{array}{l}\text { Ambos } \\
\text { sexos }\end{array}$} & VBT & $\begin{array}{c}53,9 \\
(0,066)\end{array}$ & $\begin{array}{c}0,248 \\
(0,001)\end{array}$ & $\begin{array}{l}-1,712 \\
(0,016)\end{array}$ & & & 0,90 & 206927 & \\
\hline & VBG & $\begin{array}{c}60,6 \\
(0,419)\end{array}$ & $\begin{array}{c}0,087 \\
(0,004)\end{array}$ & $\begin{array}{c}0,283 \\
(0,021)\end{array}$ & & $\begin{array}{c}0,306 \\
(0,005)\end{array}$ & 0,91 & 198431 & \\
\hline & GOMP & $\begin{array}{c}52,8 \\
(0,055)\end{array}$ & $\begin{array}{c}0,320 \\
(0,002)\end{array}$ & & $\begin{array}{l}-0,199 \\
(0,009)\end{array}$ & & 0,90 & 216410 & \\
\hline
\end{tabular}

el más empleado en la representación del crecimiento en longitud de peces, se ha determinado éste para representar el crecimiento de $M$. australis. La longitud a la edad estimada por los respectivos métodos hasta la edad 18, se muestra en la Tabla 5 y en la Fig. 4 se representan las curvas de crecimiento de machos, hembras y ambos sexos.

La comparación de parámetros de crecimiento entre sexos mediante el test multivariado $\mathrm{T}^{2}$ de Hotelling señala para este estadístico un valor de 2.829 , bastante mayor que el valor crítico $\mathrm{T}_{0}{ }_{0}=25,6$ para un nivel $\alpha=0,05$ lo que significa, la no aceptación de la hipótesis nula $\delta=0$; esto es, el conjunto de parámetros de crecimiento de machos y hembras son significativamente diferentes.

Por otra parte, el resultado del análisis SCR, también indica que las muestras comparadas originan estimados de crecimiento estadísticamente diferentes, como se presenta en la Tabla 6.

Por lo tanto, la comparación de las curvas de crecimiento mediante los dos métodos aplicados entrega como resultado la existencia de crecimiento diferente entre los sexos de $M$. australis, siendo mayor en las hembras.

En la estimación de crecimiento en peso, la relación existente entre la longitud total (LT) y el peso total (W) de
Tabla 5. Edad-longitud estimada por las diferentes ecuaciones de crecimiento para M. australis (VBT, von Bertalanffy tradicional; VBG, von Bertalanffy generalizado; GOMP, Gomperzt) / Age-length calculated by different growth equation for $M$. australis (VBT, von Bertalanffy traditional; VBG von Bertalanffy generalized; GOMP, Gomperzt)

\begin{tabular}{ccccccc}
\hline & \multicolumn{3}{c}{ Hembras } & & & Machos \\
Edad (años) & VBT & VBG & GOMP & VBT & BVG & GOMP \\
\hline 1 & 26,3 & 25,6 & 26,8 & 26,3 & 25,7 & 26,7 \\
2 & 32,6 & 33,3 & 32,4 & 32,3 & 32,9 & 32,1 \\
3 & 37,5 & 38,0 & 37,3 & 36,8 & 37,3 & 36,7 \\
4 & 41,4 & 41,5 & 41,3 & 40,4 & 40,4 & 40,3 \\
5 & 44,4 & 44,2 & 44,5 & 43,1 & 42,8 & 43,2 \\
6 & 46,8 & 46,4 & 47,0 & 45,2 & 44,8 & 45,3 \\
7 & 48,7 & 48,2 & 48,9 & 46,8 & 46,4 & 46,9 \\
8 & 50,2 & 49,8 & 50,3 & 48,0 & 47,7 & 48,1 \\
9 & 51,4 & 51,2 & 51,4 & 48,9 & 48,8 & 49,0 \\
10 & 52,3 & 52,4 & 52,2 & 49,7 & 49,8 & 49,6 \\
11 & 53,0 & 53,4 & 52,8 & 50,2 & 50,7 & 50,0 \\
12 & 53,6 & 54,3 & 53,3 & 50,7 & 51,4 & 50,4 \\
13 & 54,0 & 55,1 & 53,6 & 51,0 & 52,1 & 50,6 \\
14 & 54,4 & 55,8 & 53,8 & 51,3 & 52,6 & 50,8 \\
15 & 54,7 & 56,5 & 54,0 & 51,5 & 53,1 & 50,9 \\
16 & 54,9 & 57,1 & 54,1 & 51,6 & 53,6 & 51,0 \\
17 & 55,1 & 57,6 & 54,2 & 51,7 & 53,9 & 51,0 \\
18 & 55,2 & 58,1 & 54,3 & 51,8 & 54,3 & 51,1 \\
\hline
\end{tabular}


Tabla 6. Resultado de suma de cuadrados residuales (SCR) para machos, hembras, ambos sexos y muestra total de M. australis / Result of residual square sum (SCR) for males, females, both sexes and total sample of $M$. australis

\begin{tabular}{lr}
\hline Categorías & \multicolumn{1}{c}{ SCR } \\
\hline SCR de machos & 102508 \\
SCR de hembras & 84838 \\
SCR $s$ de machos + hembras & 187346 \\
SCR $p$ de la muestra total & 206927 \\
F calculado & 1117 \\
$F_{3(K-1) ; N-3 K,(\alpha=5 \%)}$ & \\
\hline
\end{tabular}

los ejemplares de merluza de tres aletas, por sexo, quedó representada por las funciones potenciales siguientes:

$$
\begin{array}{ll}
\text { Hembras: } & \mathrm{W}=0,01520 * \mathrm{LT}^{2,74881} \\
\text { Machos: } & \mathrm{W}=0,03993 * \mathrm{LT}^{3,10268} \\
\text { Total: } & \mathrm{W}=0,01520 * \mathrm{LT}^{2,78375}
\end{array}
$$

Los pesos asintóticos estimados a partir de la relación longitud-peso y la longitud asintótica en cada caso, entregan las siguientes ecuaciones que representan el crecimiento en peso para merluza de tres aletas (Fig. 5):

$$
\begin{array}{ll}
\text { Hembras: } & \mathrm{Wt}=933,5 \mathrm{~g}\left[1-\mathrm{e}^{-0,23902(t+1,67890)}\right]^{2,74881} \\
\text { Machos: } & \mathrm{Wt}=847,9 \mathrm{~g}\left[1-\mathrm{e}^{-0,26212(t+1,68524}\right]^{3,10268} \\
\text { Total: } & \mathrm{Wt}=877,2 \mathrm{~g}\left[1-\mathrm{e}^{-0,2470(t+1,71253}\right]^{2,78354}
\end{array}
$$

\section{Mortalidad natural}

Los valores promedios de $\mathrm{M}$, determinados por los diferentes métodos, fluctuaron entre $0,29 \mathrm{año}^{-1}$ y 0,38 año $o^{-1}$ para hembras y entre 0,28 año $^{-1}$ y 0,42 año $^{-1}$ para machos, siendo el error estándar menor en la estimación por el método de Pauly. El valor de mortalidad natural ponderado por el inverso de la varianza fue $0,320 \mathrm{año}^{-1}$ para las hembras y para los machos de 0,336 año $^{-1}$ (Tabla 7).

\section{Discusión}

En diferentes estudios de la edad en Micromesistius australis, se ha señalado que el otolito sagitta ha resultado muy apropiado para la determinación de la edad. Payá (1997) informa la formación de un anillo hialino y un opaco anual con un máximo en septiembre para el primero. Por

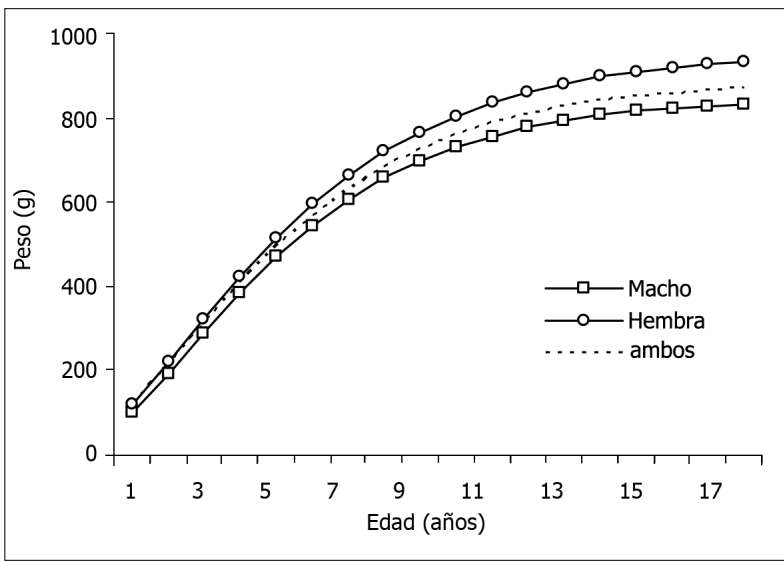

Figura 5. Curvas de crecimiento en peso ajustadas para $M$. australis / Growth curves in weight fitted for M. australis

otra parte, Ojeda et al. (1998) y sobre la base de información de lecturas de otolitos para el periodo 1990-1996, seleccionó sólo los años 1991 y 1994 como los más completos para validar la formación de los anillos, encontrando que los mayores valores de deposición del anillo hialino fueron entre mayo y agosto con un máximo en agosto. En el presente estudio, que consiste en una revisión del anterior, pero considerando el periodo entre 1991 y 1994, por una mayor cantidad de muestras mensuales, se determinó que el periodo de mayor formación del hialino fue entre junio y agosto con un máximo en julio. Esta diferencia es mínima y estaría condicionada por la cantidad de observaciones de cada periodo.

El número máximo de anillos hialinos observados, equivalentes a igual número de edades (años) fue 18 , tanto en otolitos de machos como de hembras, pero con muy baja representación para el anillo 14 y mayores. Por tanto, el mayor anillo y mejor representado fue el 13 (219 y 239 lecturas en machos y hembras, respectivamente), lo que en general coincide con lo registrado por Payá (1997) ${ }^{1}$ y Ojeda et al. (1998).

El crecimiento de $M$. australis ha sido analizado por distintos autores para diferentes áreas geográficas del Pacífico suroriental, el Atlántico suroccidental y aguas de Nueva Zelanda (Tabla 8). En Chile, el ajuste de los parámetros de crecimiento de von Bertalanffy se ha realizado mediante el ajuste no lineal de la longitud-edad. En el estudio de Payá (1997) los valores de $\mathrm{L}_{\infty}(55,3 \mathrm{~cm}$ machos y $56,4 \mathrm{~cm}$ hembras) son algo mayores a los

\footnotetext{
${ }^{1}$ Payá I. 1997. Investigación CTP de merluza de tres aletas 1997. Informe Final. Informe Técnico, 31 pp. Instituto de Fomento Pesquero, Chile
} 
Tabla 7. Mortalidad natural estimada por métodos bioanalógicos para cada sexo de M. australis y estimación de M ponderada (1. Pauly 1980, 2. Alverson \& Cartney 1975, 3. Höening 1983 y 4. Taylor 1960) / Natural mortality estimated by bioanalogical methods for each sex of M. australis and estimation of ponderated M (1. Pauly 1980, 2. Alverson \& Cartney 1975, 3. Höening 1983 and 4. Taylor 1960).

\begin{tabular}{|c|c|c|c|c|c|c|c|c|c|c|c|c|}
\hline \multirow[b]{2}{*}{ Métodos } & \multicolumn{4}{|c|}{ Hembras } & \multicolumn{4}{|c|}{ Machos } & \multicolumn{4}{|c|}{ Ambos } \\
\hline & 1 & 2 & 3 & 4 & 1 & 2 & 3 & 4 & 1 & 2 & 3 & 4 \\
\hline M prom. & 0,310 & 0,317 & 0,368 & 0,303 & 0,335 & 0,292 & 0,410 & 0,342 & 0,320 & 0,308 & 0,384 & 3,318 \\
\hline Error estándar & 0,0016 & 0,0020 & 0,0034 & 0,0030 & 0,0017 & 0,0022 & 0,0039 & 0,0036 & 0,3224 & 0,3044 & 0,3883 & 0,32194 \\
\hline Límite inferior & 0,305 & 0,310 & 0,357 & 0,294 & 0,330 & 0,284 & 0,398 & 0,331 & 0,315 & 0,305 & 0,380 & 0,314 \\
\hline Límite superior & 0,315 & 0,323 & 0,379 & 0,314 & 0,341 & 0,299 & 0,423 & 0,354 & 0,325 & 0,311 & 0,390 & 0,324 \\
\hline M ponderada & \multicolumn{4}{|c|}{0,320} & \multicolumn{4}{|c|}{0,336} & \multicolumn{4}{|c|}{0,327} \\
\hline
\end{tabular}

Tabla 8. Valores de los parámetros de crecimiento en longitud estimados para M. australis, por diferentes autores. (1) Atlántico Sudoccidental; (2) Pacífico suroriental; (3) Nueva Zelanda / Value of the growth parameter in length estimated for M. australis by different authors. (1) Southwest Atlantic; (2) Southeast Pacific; (3) New Zealand

\begin{tabular}{|c|c|c|c|c|c|c|}
\hline Sexo & $\mathrm{L}_{\infty}$ & K & $\mathrm{t}_{0}$ & Fuente & $\begin{array}{l}\text { Estructura } \\
\text { observada }\end{array}$ & $\begin{array}{c}\text { Información } \\
\text { utilizada }\end{array}$ \\
\hline M & 56,9 & 0,1980 & $-1,840$ & Barrera-Oro \& & Secciones & Edad-longitud \\
\hline $\mathrm{H}$ & 60,1 & 0,1770 & $-2,070$ & Tomo 1988 (1) & delgadas & retrocalculada \\
\hline M & 54,8 & 0,2781 & $-1,258$ & \multirow[t]{2}{*}{ Cassia 2000 (1) } & Secciones & \multirow{2}{*}{$\begin{array}{l}\text { Edad-longitud } \\
\text { actual }\end{array}$} \\
\hline $\mathrm{H}$ & 59,7 & 0,2168 & $-1,429$ & & transversales & \\
\hline M & 55,3 & 0,2330 & $-1,460$ & \multirow[t]{2}{*}{ Payá 1997 (2) } & Otolito entero & \multirow{2}{*}{$\begin{array}{l}\text { Edad-longitud } \\
\text { retrocalculada }\end{array}$} \\
\hline $\mathrm{H}$ & 56,4 & 0,2260 & $-1,164$ & & pulido & \\
\hline M & 46,5 & 0,3900 & $-0,680$ & \multirow{2}{*}{$\begin{array}{c}\text { Hanchet \& Uozomi } \\
1996(3)\end{array}$} & Secciones & \multirow{2}{*}{$\begin{array}{l}\text { Edad-longitud } \\
\text { (horquilla) actual }\end{array}$} \\
\hline $\mathrm{H}$ & 50,1 & 0,3500 & $-0,710$ & & delgadas & \\
\hline M & 46,7 & 0,3600 & $-0,010$ & \multirow{2}{*}{$\begin{array}{c}\text { Hanchet \& Uozomi } \\
1996 \text { (3) }\end{array}$} & \multirow[t]{2}{*}{ Multifan } & \multirow{2}{*}{$\begin{array}{c}\text { Frecuencias de } \\
\text { longitud (horquilla) }\end{array}$} \\
\hline $\mathrm{H}$ & 49,4 & 0,4000 & 0,190 & & & \\
\hline M & 51,5 & 0,2677 & $-1,593$ & \multirow[t]{2}{*}{ Ojeda et al. 1998 (2) } & \multirow{2}{*}{$\begin{array}{l}\text { Otolito entero } \\
\text { pulido }\end{array}$} & \multirow{2}{*}{$\begin{array}{l}\text { Edad-longitud } \\
\text { retrocalculada }\end{array}$} \\
\hline $\mathrm{H}$ & 55,2 & 0,2465 & $-1,469$ & & & \\
\hline M & 55,0 & 0,2551 & 1,8871 & \multirow[t]{2}{*}{ Ojeda et al. 1998 (2) } & \multirow{2}{*}{$\begin{array}{l}\text { Otolito entero } \\
\text { pulido }\end{array}$} & \multirow{2}{*}{$\begin{array}{l}\text { Edad-longitud } \\
\text { actual }\end{array}$} \\
\hline $\mathrm{H}$ & 59,1 & 0,2388 & 1,6109 & & & \\
\hline M & 52,0 & 0,1800 & $-3,3960$ & \multirow[t]{2}{*}{ Ojeda et al. 1998 (2) } & \multirow{2}{*}{$\begin{array}{l}\text { Otolito entero } \\
\text { pulido }\end{array}$} & \multirow{2}{*}{$\begin{array}{c}\text { Edad-longitud } \\
\text { actual }+ \text { retrocalculada }\end{array}$} \\
\hline $\mathrm{H}$ & 55,9 & 0,1680 & $-3,2620$ & & & \\
\hline M & 52,1 & 0,2621 & $-1,6852$ & \multirow[t]{2}{*}{ Actual estudio (2) } & \multirow{2}{*}{$\begin{array}{l}\text { Otolito entero } \\
\text { pulido }\end{array}$} & \multirow{2}{*}{$\begin{array}{l}\text { Edad-longitud } \\
\text { retrocalculada }\end{array}$} \\
\hline $\mathrm{H}$ & 55,7 & 0,2390 & $-1,6789$ & & & \\
\hline
\end{tabular}


Tabla 9. Comparación de estimaciones de M para M. australis (Ojeda et al. 1998 y estudio actual; valores de las varianzas se indican entre paréntesis / Comparison of M estimation for M. australis (Ojeda et al. 1998 and present study; variance values in parentheses)

\begin{tabular}{lcccccc}
\hline & \multicolumn{2}{c}{ M promedio } & & & M ponderado \\
& Membra & Ambos sexos & Macho & Hembra & Ambos sexos \\
\hline Ojeda et al. 1998 & $0,2284-0,5188$ & $0,2331-0,4638$ & - & 0,4425 & 0,3917 & - \\
& $(6,9 \mathrm{E}-07-6,6 \mathrm{E}-06)$ & $(7,5 \mathrm{E}-07-1,0 \mathrm{E}-05)$ & & & 0,3200 & 0,3270 \\
Estudio actual & $0,2920-0,4100$ & $0,3030-0,3680$ & $0,3080-03840$ & 0,3360 & 0 & \\
& $(5,1 \mathrm{E}-06-1,5 \mathrm{E}-05)$ & $(3,1 \mathrm{E}-03-3,4 \mathrm{E}-03)$ & $(3 \mathrm{E}-01-3,9 \mathrm{E}-01)$ & & & \\
\hline
\end{tabular}

estimados en el estudio actual (machos $52,1 \mathrm{~cm}$ y hembra $55,7 \mathrm{~cm})$, siendo una posible explicación el tamaño de muestra (452 machos y 474 hembras) y el rango de longitudes utilizados por dicho autor. Con respecto a los valores estimados por Ojeda et al. (1998) éstos son muy similares, ya que utilizaron información de longitud-edad retrocalculada, pero no así con los valores ajustados con información a la edad actual, donde los $\mathrm{L}_{\infty}$ (machos 55 $\mathrm{cm}$, hembras $59,1 \mathrm{~cm}$ ) son superiores por el sesgo en las edades 1 y 2 , considerando que los parámetros ajustados a partir de información combinada longitud-edad retrocalculada y edad actual, los valores de $\mathrm{L}_{\infty}$ son similares, pero los parámetros $\mathrm{K} \mathrm{y} \mathrm{t}_{0}$ difieren. En todo caso, la combinación señalada es un procedimiento incorrecto, ya que se mezcla información de dos métodos diferentes.

Por otra parte, los valores de los parámetros de crecimiento en longitud estimados en el actual estudio difieren de los informados por Barrera \& Tomo (1988), Cassia (2000) y Hanchet \& Uozomi (1996). Respecto a los dos primeros, las diferencias se explican porque Micromesistius australis, presente en el O. Pacífico y O. Atlántico, pertenecerían a stocks diferentes de acuerdo con un análisis de indicadores de segregación ecológica, tales como composición por longitud, edad de primera madurez sexual, morfometría de otolitos, composición parasitaria, composición de microelementos e isótopos estables en otolitos (Niklischek et al. 2009, Arkhipin et al. 2009). Esto posiblemente también sería la causa de las diferencias halladas en relación a los resultados obtenidos por Hanchet \& Uozomi (1996) en aguas de Nueva Zelanda, además estos autores utilizaron la longitud horquilla en vez de la longitud total (considerada por los otros autores).

En las estimaciones de mortalidad natural se excluyó el método de Alagaraja (1984), ya que en los estimados de Ojeda et al. (1998) resultaron bastante más elevadas que los otros métodos (Tabla 9), lo que se explicaría al incorporar en la ecuación un valor de edad máxima subestimada, lo que incide en el valor de M, considerando que el autor definió en forma provisional la longevidad de las especies como la edad a la que el $99 \%$ de una cohorte habría muerto si hubiese estado expuesta sólo a mortalidad natural. En síntesis, el considerar los valores estimados por este método ha incidido finalmente en los estimados ponderados de $\mathrm{M}$ por sexo que presentan mayores valores que los estimados actuales (Tabla 9).

Se concluye que en los otolitos de $M$. australis se forman claramente anillos hialinos y opacos en forma alternada con la depositacion de los primeros entre junio-agosto. Las hembras presentan un crecimiento significativamente mayor a los machos a la misma edad. El mejor ajuste del crecimiento en longitud se obtuvo mediante el modelo de von Bertanlanffy tradicional utilizando los valores edadlongitud determinados a través del retrocalculo.

\section{Agradecimientos}

Los autores agradecen al Fondo de Investigación Pesquera por autorizar el uso de los datos obtenidos a través del proyecto FIP 97-15, cuyos autores participaron en su desarrollo. Hacemos extensivos nuestros agradeci mientos a tres revisores anónimos por sus acertadas observaciones.

\section{LiteratURA CITADA}

Aguayo M, Z Young, $R$ Bustos, V Ojeda, T Peñailillo, $R$ Gili, C Vera \& H Robotham. 1986. Diagnóstico de las principales pesquerías nacionales demersales (peces) zona sur-austral. 1985. Estado de Situación del Recurso. Instituto de Fomento Pesquero, AP 86/55: 1-143.

Aguayo M, Z Young, R Bustos, T Peñailillo, V Ojeda, C Vera, H Hidalgo \& I Céspedes. 1987. Diagnóstico de las principales pesquerías nacionales demersales (peces) zona sur-austral. 1986. Estado de Situación del Recurso. Instituto de Fomento Pesquero, AP 87/3: 1-209.

Aguayo M, I Payá, R Bustos, V Ojeda, R Gili, C Vera, I Céspedes \& L Cid. 1990. Diagnóstico de las principales 
pesquerías nacionales demersales (peces) zona sur-austral. 1988. Estado de Situación del Recurso. Instituto de Fomento Pesquero, AP 89/17: 1-161.

Aguayo M, I Payá, C Vera, V Ojeda, I Céspedes, J Donoso, A Zuleta, R Bustos, L Cid \& L Muñoz. 1991. Diagnóstico de las principales pesquerías nacionales demersales (peces) zona sur-austral. 1990. Estado de Situación del Recurso. Instituto de Fomento Pesquero, SGI 91/6: 1-83.

Akaike H. 1974. A new look at the statistical model identification. IEEE Transaction on Automatic Control 19(6): 716-723.

Alagaraja K. 1984. Simple method for estimation of parameters for assessing exploited fish stocks. Indian Journal of Fisheries 31: 177-208.

Alverson DL \& MJ Carney. 1975. A graphic review of the growth and decay of population cohorts. Journal du Conseil International pour l'Exploration de la Mer 36: 133-143.

Angelescu V, F Gneri \& A Nani. 1958. La merluza del mar argentino (biología y taxonomía), 224 pp. Secretaría de Marina, Servicio de Hidrografía Naval, Buenos Aires.

Araya M \& L Cubillos. 2002. El análisis retrospectivo del crecimiento en peces y sus problemas asociados. Gayana 66(2): 161-179.

Arkhipin AI, PC Scuchert \& L Danyushevsky. 2009. Otolith chemistry reveals fine population structure and close affinity total Pacific and Atlantic oceanic spawning ground in the migratory southern blue whiting (Micromesistius australis australis). Fisheries Research 96(2-3): 188-194.

Avilés S, M Aguayo, F Hinostroza \& J Cañón. 1979. Merluza de tres aletas. Estado actual de las principales pesquerías nacionales. Bases para un desarrollo pesquero. Peces. Instituto de Fomento Pesquero, AP 79/18: 1-25.

Barrera-Oro E \& A Tomo. 1988. New information on age and growth in length of Micromesistius australis, Norman 1937 (Pisces, Gadidae), in the South-West Atlantic. Polar Biology 8: 341-351.

Bernard R. 1981. Multivariate analysis as a mean of comparing growth in fish. Canadian Journal of Fisheries and Aquatic Sciences 38: 233-236.

Beverton R \& S Holt. 1957. On the dynamic of exploited fish populations. Fishery Investigation Series II 19: 1-533.

Cassia MC. 2000. Age and growth of the southern blue whiting Micromesistius australis in the SW Atlantic. Scientia Marina 64(3): 269-274.

Cerrato RM. 1990. Interpretable statistical test for growth comparisons using parameters in the von Bertalanffy equation. Canadian Journal of Fisheries and Aquatic Sciences 47: 14161426.

Chen Y, A Jackson \& H Harvey. 1992. A comparison of von Bertalanffy and polynomial functions in modeling fish growth data. Canadian Journal of Fisheries and Aquatic Sciences 49: 1228-1235.
Chilton ED \& RJ Beamish. 1982. Age determination methods for fishes studied by the Groundfish Program at the Pacific Biological Station. Canadian Special Publication of Fisheries and Aquatic Sciences 60: 1-102.

Chong JV, M Aguayo \& I Payá. 2007. Estimación de edad, crecimiento y mortalidad natural de la merluza de cola, Macruronus magellanicus Lönnberg, 1907 (Macruronidae, Gadiformes) en el Océano Pacífico Suroriental. Revista de Biología Marina y Oceanografía 42(3): 311-333.

Chugunova NI. 1963. Age and growth studies in fish. A systematic guide for ichthyologists, $132 \mathrm{pp}$. National Science Foundation \& The Israel Program for Scientific Translations, Washington.

Cohen D, T Inada, T Iwamoto \& N Scialabba. 1990. Gadiform fishes of the world (Order Gadiformes). FAO Species Catalogue. FAO Fisheries Synopsis 125(10): 1-442.

Csirke J. 1980. Introducción a la dinámica de poblaciones de peces. FAO Documento Técnico de Pesca 192: 1-82.

Dannevig A. 1933. On the age growth of the Cod (Gadus callarias L.) from the Norwegian Skagerrack Coast. Report on Norwegian Fisheries and Marine Investigations 4(1): 1-145.

Efron B. 1985. Bootstrap confidence imtervals for a class of parametric problems. Biometrika 72(1): 45-48.

Francis RIC. 1990. Back-calculation of fish length: a critical review. Journal of Fish Biology 36: 883-902.

Hanchet S \& Y Uozumi. 1996. Age validation and growth of southern blue whiting Micromesistius australis Norman, in New Zealand. New Zealand Journal of Marine and Freshwater Research 30: 57-67.

Höening JM. 1983. Empirical use of longevity data to estimate mortality rates. Fishery Bulletin 82: 898-902.

Lillo S, R Céspedes, V Ojeda \& A Saavedra. 2005. Evaluación hidroacústica del stock parental de merluza de tres aletas en su unidad de pesquería, año 2004 (Fase I). Informe Final, Proyecto FIP 2004-08-1: 1-104. [en línea] <http://www.fip.cl/ FIP/Archivos/pdf/informes/inffinal\%202004-08-1.pdf>

Niklitschek E, C Canales, S Ferrada, R Galleguillos, M George-Nascimento, E Hernández, C Herranz, A Lafon, R Roa \& P Toledo. 2009. Unidades poblacionales de merluza de tres aletas (Micromesistius australis). Informe Final, Proyecto FIP 2006-15: 1-108. [en línea] <http://www.fip.cl/ FIP/Archivos/pdf/informes/inffinal\%202006-15.pdf >

Ojeda V, F Cerna, M Aguayo, I Payá \& J Chong. 1998. Estudio de crecimiento y construcción de claves talla edad de merluza de tres aletas y merluza de cola. Informe Final, Proyecto FIP 97-15: 1-134. [en línea] <http://www.fip.cl/ FIP/Archivos/pdf/informes/inffinal\%2097-15.pdf>

Pauly D. 1980. On the interrelationships between natural mortality, growth parameters, and mean environmental temperature in 175 fish stock. Journal du Conseil International pour l'Exploration de la Mer 39: 175-192. 
SERNAPESCA. 2002. Anuario estadístico de pesca 2002, 151 pp. Servicio Nacional de Pesca, Valparaíso. [en línea] <http:// www.sernapesca.cl>

Sparre P \& S Venema. 1998. Introducción a la evaluación de recursos pesqueros tropicales. Parte 1- Manual. FAO Documento Técnico de Pesca 306/1 (Rev.2): 1-635.
Taylor CC. 1960. Temperature, growth and mortality of the Pacific cockle. Journal du Conseil International por l'Exploration de la Mer 26: 117-124.

Wöhler O, J Hansen, M Cassia \& H Cordo. 2007. Evaluación de polaca (Micromesistius australis) en el Atlántico Sudoccidental. Periodo 1987-2001. INIDEP, Informe Técnico 62: $27-52$.

Recibido el 24 de marzo de 2009 y aceptado el 12 de mayo de 2010 